Supporting Information

\title{
Molecular mechanism of the light-induced conformational switching of the LOV domain in aureochrome-1
}

Itsuki Kobayashi, Hiroto Nakajima and Osamu Hisatomi*

Department of Earth and Space Science, Graduate School of Science, Osaka University, Toyonaka, Osaka 560-0043, Japan

*Correspondence: hisatomi@ess.sci.osaka-u.ac.jp 


\section{The supporting information containing Figure S1-S14 and Table S1-S2}

\section{CONTENTS}

Fig. S1) Domain structure of PZ and list of oligonucleotides

Fig. S2) Spectral changes during dark regeneration of Q317 mutants

Fig. S3) The $R_{H(\text { app })}$ against protein concentrations of Q317 mutants

Fig. S4) Spectral changes and the $R_{H(a p p)}$ of Q317N mutant at $5{ }^{\circ} \mathrm{C}$

Fig. S5) Calibration of MW for SEC measurements

Fig. S6) Correlation between $R_{H}$ and calculated molecular weight of Q317 mutants

Fig. S7) EMSA data of Q317 mutants for AUREO1-specific oligonucleotide

Fig. S8) $E C_{50}$ values of $\mathrm{PZ}$ mutants for non-specific oligonucleotide

Fig. S9) QCM data of Q317 mutants for AUREO1-specific oligonucleotide

Fig. S10) Spectral changes during dark regeneration of F298 and F298/Q317 mutants

Fig. S11) The $R_{H(a p p)}$ against protein concentrations of F298 and F298/Q317 mutants

Fig. S12) Correlation between $R_{H}$ and calculated molecular weight of F298 and F298/Q317 mutants

Fig. S13) EMSA data of F298 and F298/Q317 mutants for AUREO1-specific oligonucleotide

Fig. S14) QCM data of F298 and F298/Q317 mutants for AUREO1-specific oligonucleotide

Table S1) The peak elution volumes and apparent MW of Q317 mutants

Table S2) The peak elution volumes and apparent MW of F298 and F298/Q317 mutants 
(A)

$113123 \quad 144$
$M \quad \mathbf{G} \quad$ bZIP
178 218 $320 \quad 348$

(B)

\begin{tabular}{|c|c|c|}
\hline PZ_F298L-F & 5' - AACCTGCTGTTCGTGGCTGGCCTGCGC & $-3^{\prime}$ \\
\hline PZ_F298L-R & 5' - CACGAACAGCAGGTTCCAAAAAGTGGT & $-3^{\prime}$ \\
\hline PZ_F298V-F & 5' - GAACCTGGTTTTCGTGGCTGGCCTGC & \\
\hline PZ_F298V-R & 5' - ACGAAAACCAGGTTCCAAAAAGTGGT & -3 \\
\hline PZ_Q317S-F & 5' - GGCGTTAGTAGTAAAGTCTCCGAAGAC & $-3^{\prime}$ \\
\hline PZ_Q317S-R & $5^{\prime}$ - TTTACTACTAACGCCCACGTAGTTAAC & $-3^{\prime}$ \\
\hline PZ_Q317E-F & 5' - GGCGTTGAAAGTAAAGTCTCCGAAGAC & \\
\hline PZ_Q317E-R & 5' - TTTACTTTCAACGCCCACGTAGTTAAC & \\
\hline PZ_Q317V-F & 5' - GGCGTTGTGAGTAAAGTCTCCGAAGA & \\
\hline PZ_Q317V-R & $5^{\prime}$ - TTTACTCACAACGCCCACGTAGTTAAC & $-3^{\prime}$ \\
\hline PZ_Q317N-F & 5' - GGCGTTAACAGTAAAGTCTCCGAAGAC & $-3^{\prime}$ \\
\hline PZ_Q317N-R & $5^{\prime}$ - TTTACTGTTAACGCCCACGTAGTTAAC & \\
\hline PZ_Q317L-F & 5' - GGCGTTCTGAGTAAAGTCTCCGAAGA & \\
\hline PZ_Q317L-R & 5' - TTTACTCAGAACGCCCACGTAGTTAA & 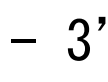 \\
\hline Apo & 5' - GCTGTCTGACGTCAGACAGC -3' & \\
\hline Cpo & 5' - GCTGTGCAGATCTGCACAGC -3' & \\
\hline
\end{tabular}

Fig S1 (A) The domain structure of PZ, (B) nucleotide sequences of primers used for site-directed mutagenesis, and (C) nucleotide sequences of oligonucleotides used for binding assays. Underlined sequences indicates the target sequence of VfAUREO1. ${ }^{8}$ 

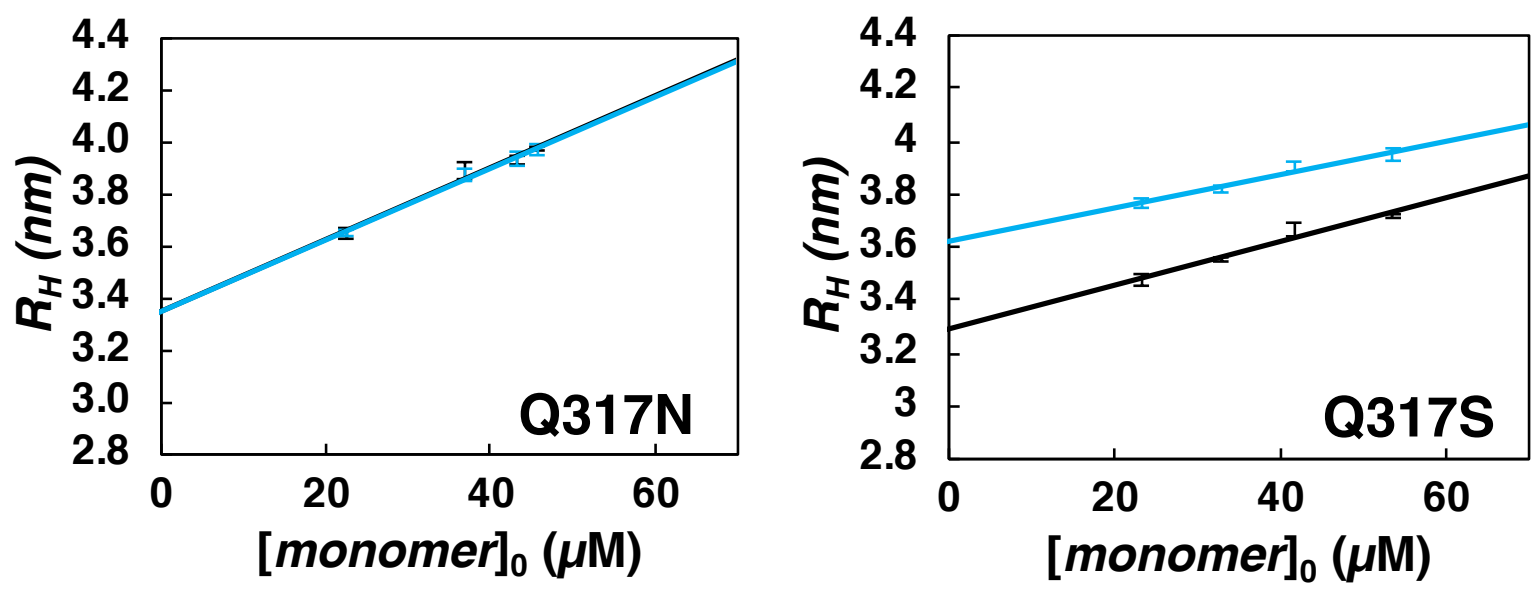

(C)
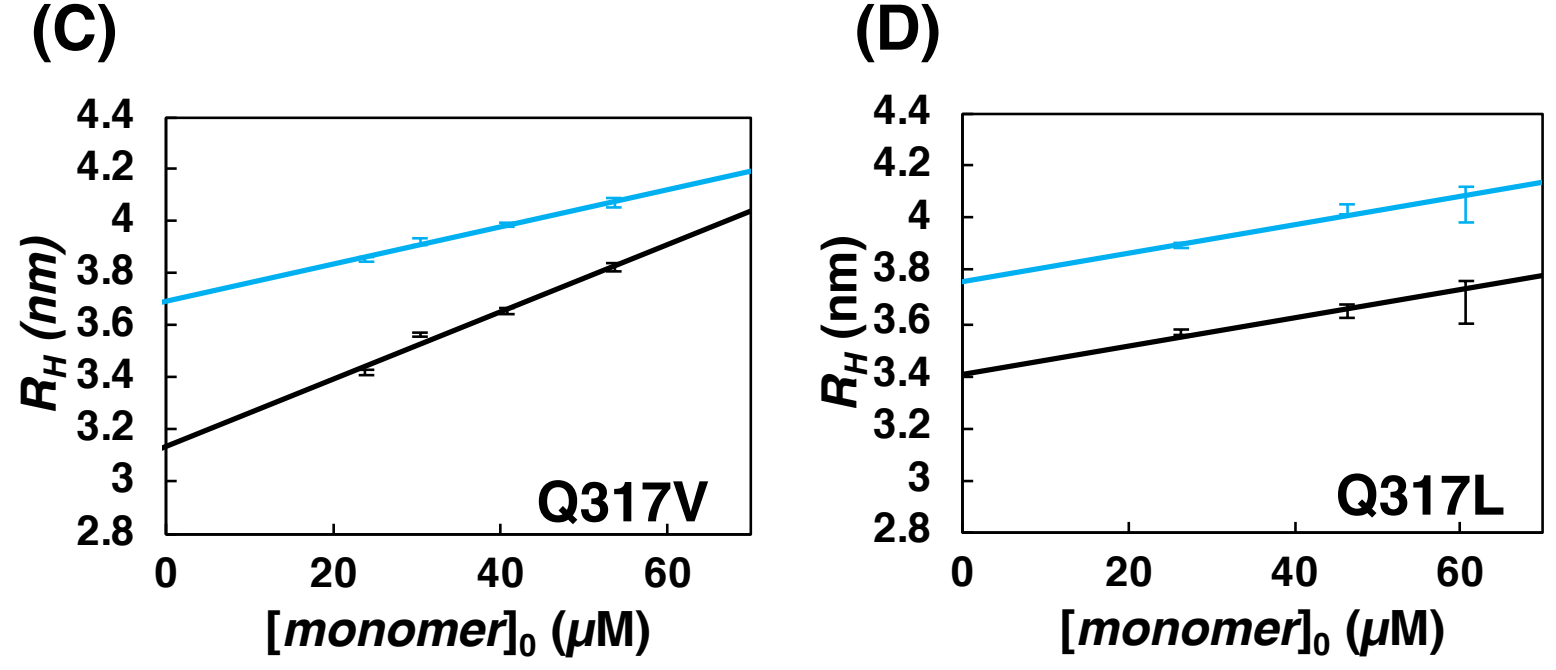

Fig. S3 $R_{H(a p p)}$ according to protein concentration relative to [monomer $]_{0}$ of (A) Q317N, (B) Q317S, (C) Q317V, and (D) Q317L. Black and blue bars and lines represent the $R_{H(a p p)}$ at each concentration with standard deviation $(\mathrm{SD}, \mathrm{n} \geq 6)$ and regression line in the $\mathrm{D}$ and L states, respectively. 
(A)

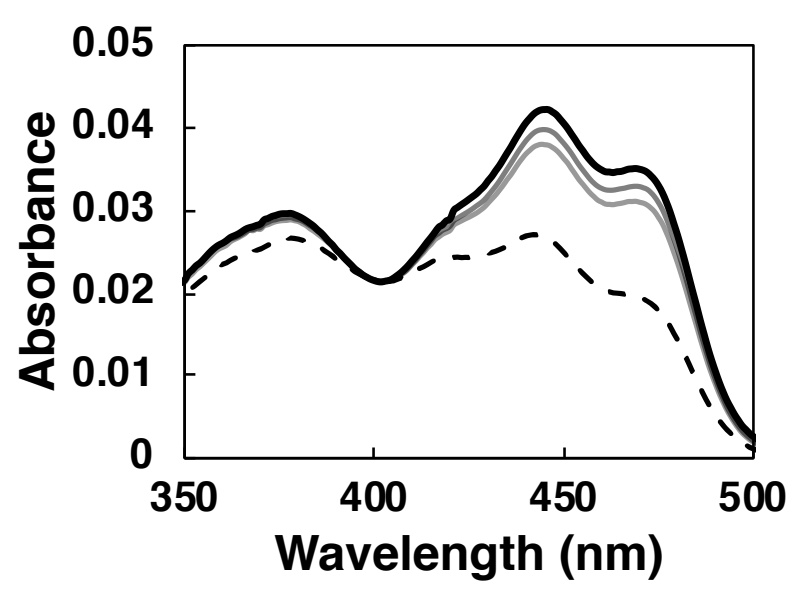

(B)

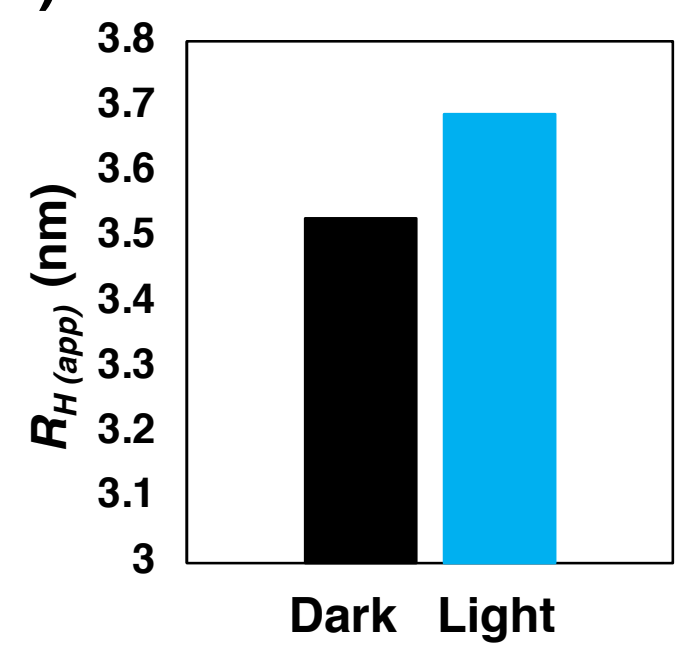

Fig. S4 (A) Spectral change during dark regeneration and (B) the $R_{H \text { (app) }}$ of Q317N at $5{ }^{\circ} \mathrm{C}$. 
(A)

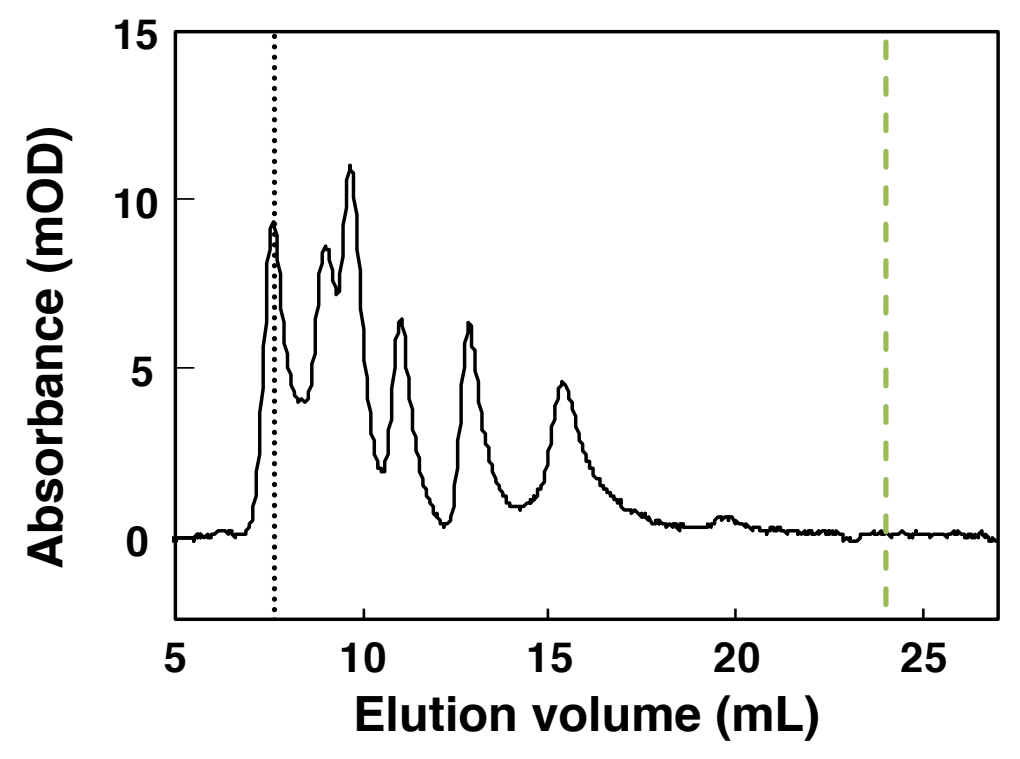

(B)

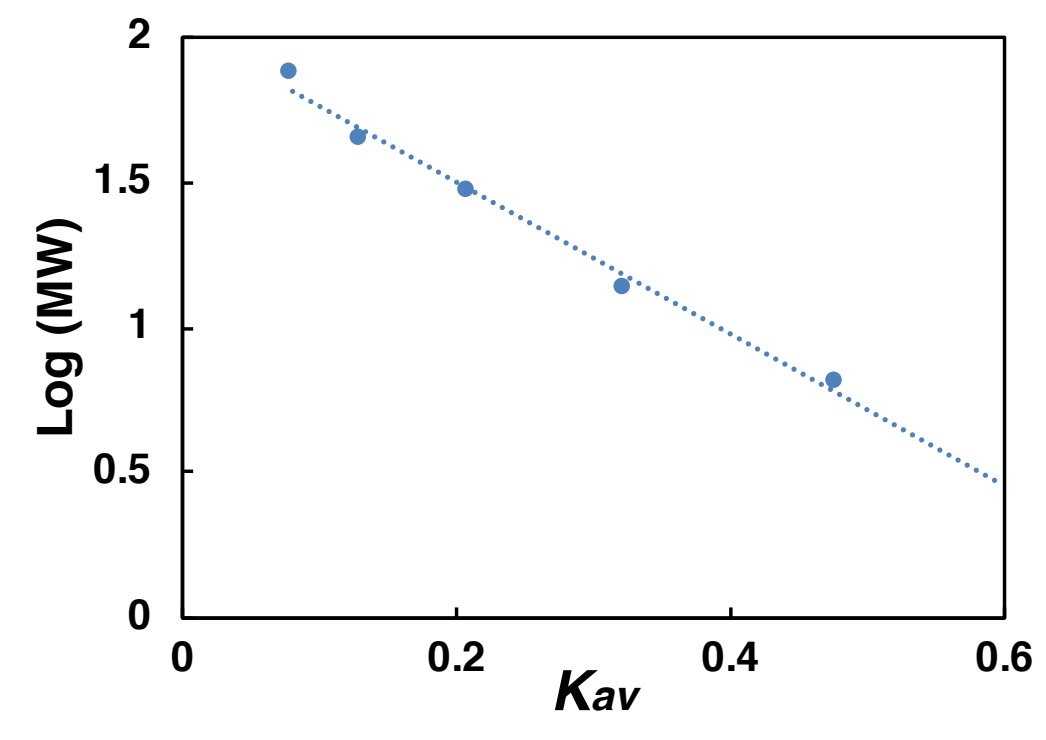

Fig. S5 Calibration of molecular weight (MW) for SEC measurements. (A) Elution profile of marker proteins; blue dextran $(2,000 \mathrm{kDa})$, conalbumin $(75 \mathrm{kDa})$, ovalbumin $(44 \mathrm{kDa})$, carbonic anhydrase $(29 \mathrm{kDa})$, ribonuclease $\mathrm{A}(13.7 \mathrm{kDa})$, and aprotinin $(6.5 \mathrm{kDa})$. (B) Relationship between MW and Kav. The regression line represents the relationship of $\log _{10}(\mathrm{MW})=-2.615 \mathrm{Kav}+2.024$ 
(A)

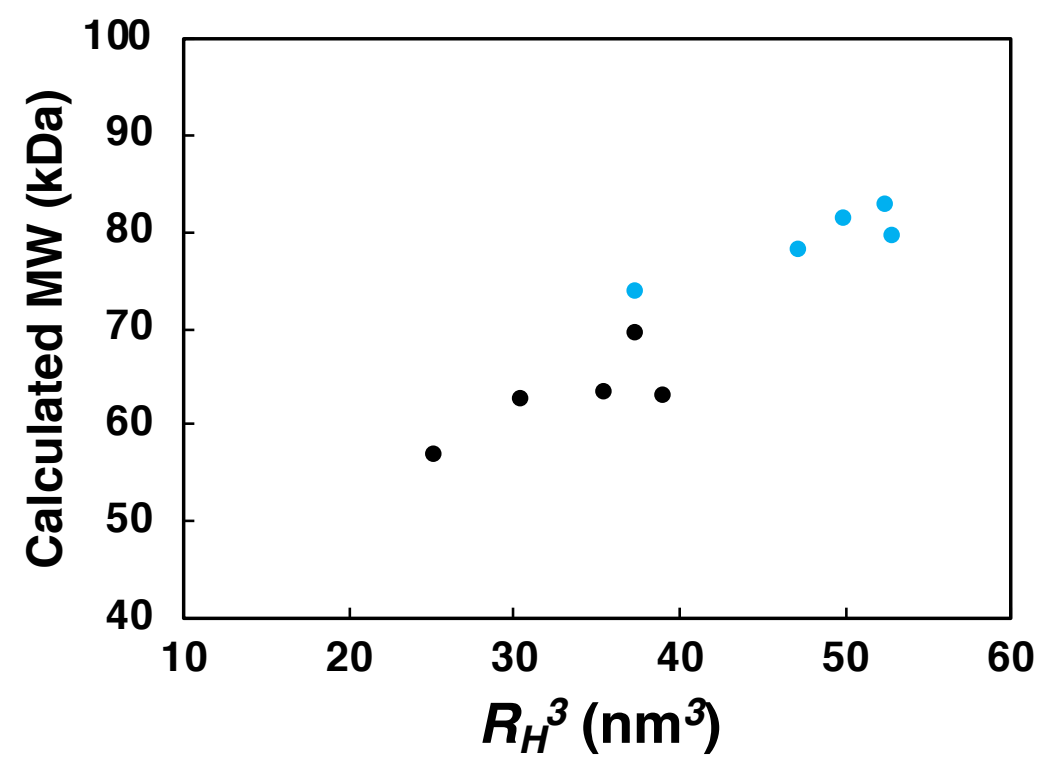

(B)

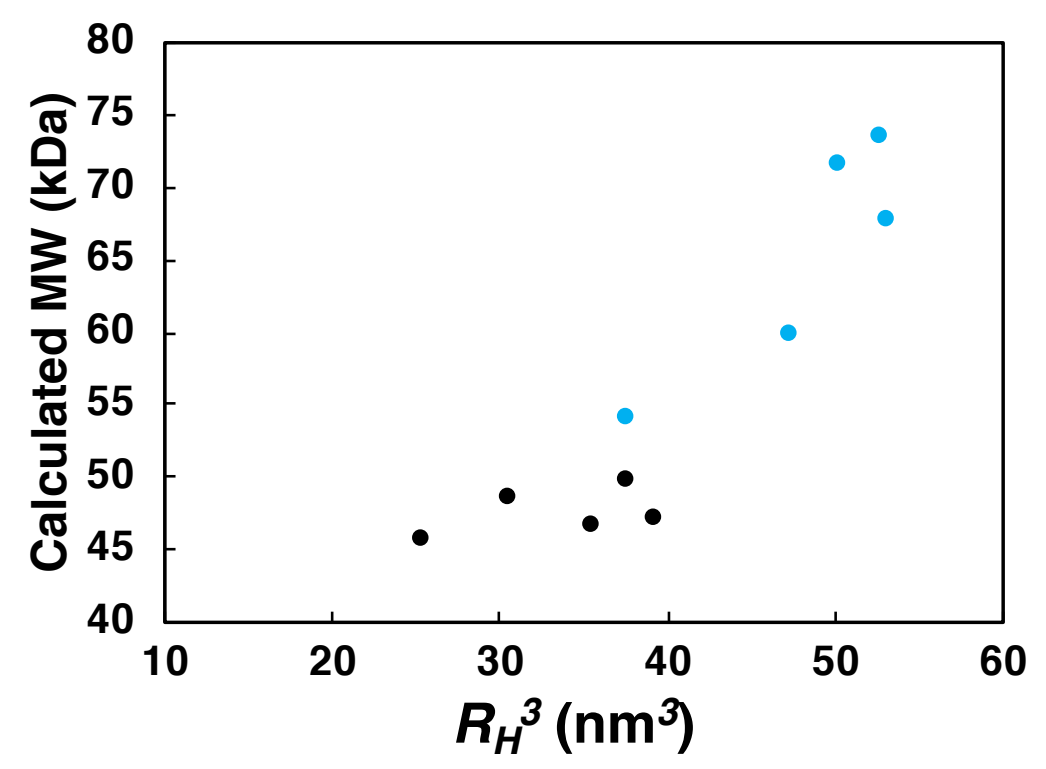

Fig. S6 Correlation between $R_{H}^{3}$ and calculated molecular weight (MW) from peak elution volumes of $\mathrm{Q} 317$ mutants using equation for globular proteins. ${ }^{23}$ Injected protein concentrations are (A) $100 \mu \mathrm{M}$ and (B) $8 \mu \mathrm{M}$. Black and blue symbols represent data in the D and $\mathrm{L}$ states, respectively. 


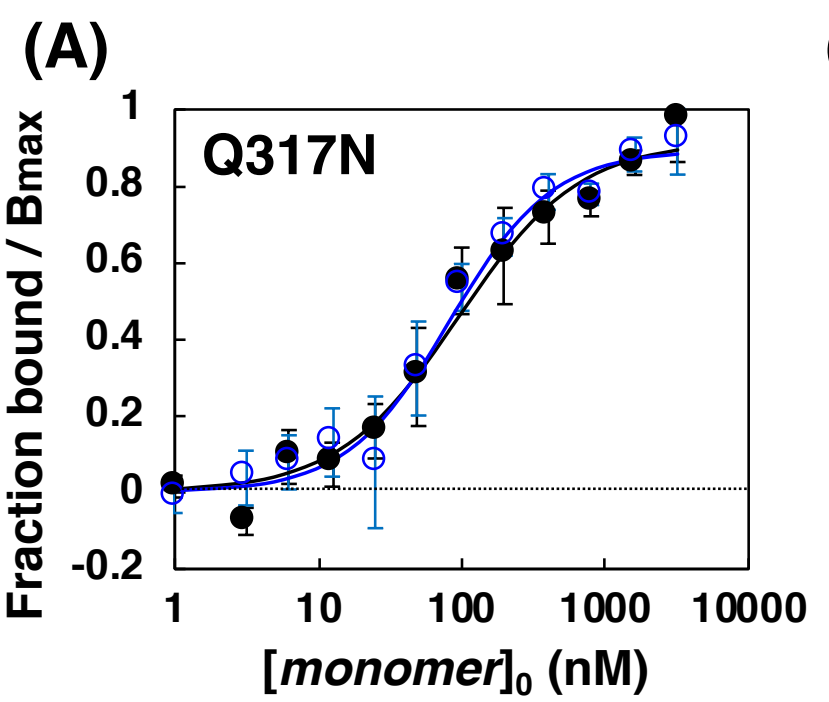

(B)

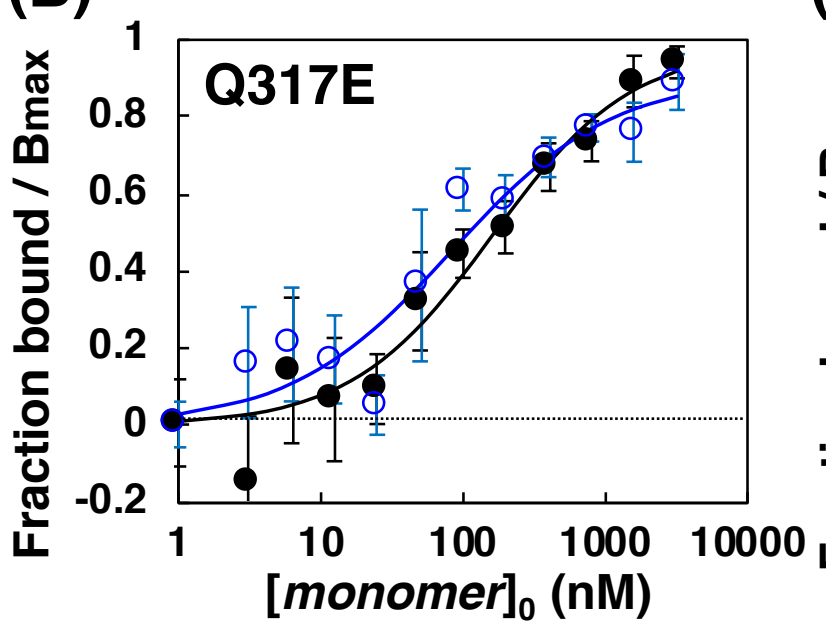

(C)

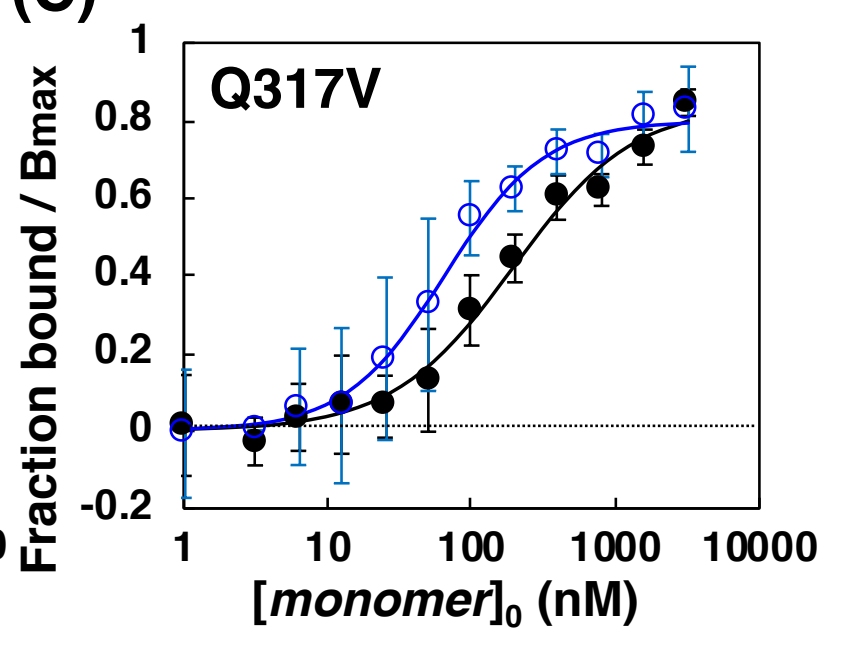

(D)

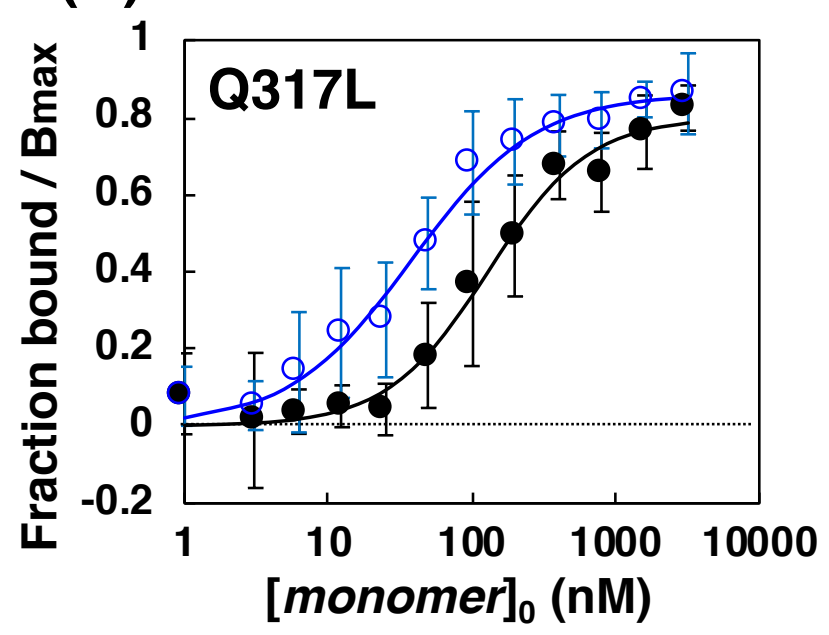

(E)

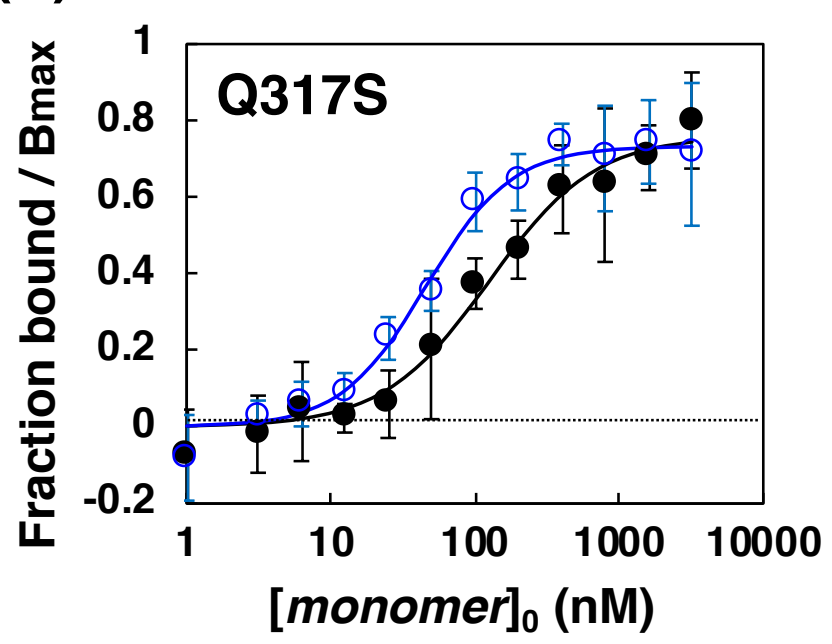

Fig. S7 The normalized dsApo fractions bound to Q317 mutants plotted against protein concentration [monomer $]_{0}$ with fitting curves in the $\mathrm{D}$ (filled black circles and black lines) and $\mathrm{L}$ (open blue circles and blue lines) states for (A) Q317N, (B) Q317E, (C) Q317S, (D) Q317V, and (E) Q317L. Bars represent SDs $(n \geq 4)$. 


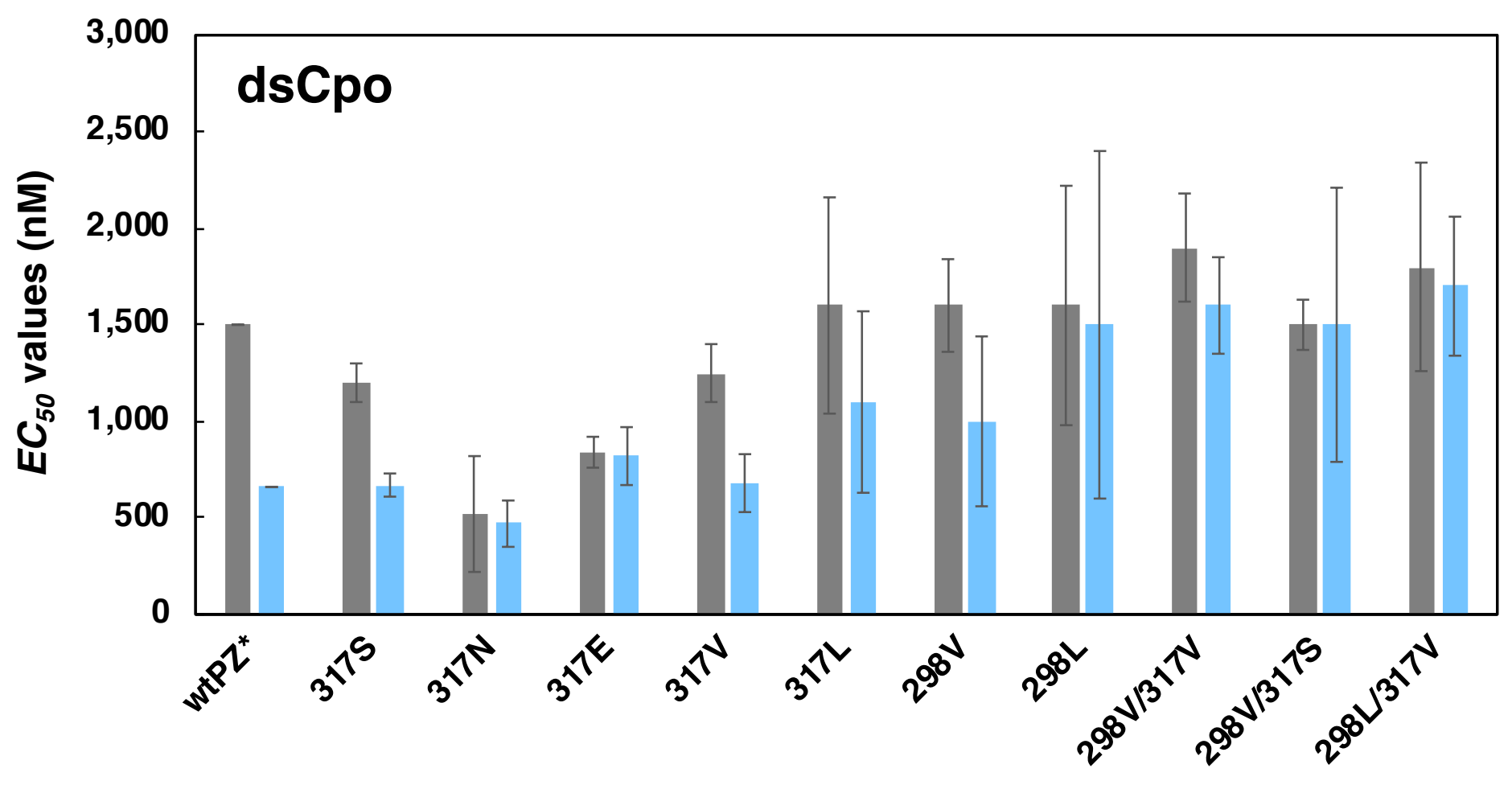

Fig. S8 The $E C_{50}$ values of wtPZ and mutants for a control (nonspecific) palindromic oligonucleotide (dsCpo) according to EMSA in the D (gray) and L (blue) states. Bars represent SDs $(n \geq 3)$. Data for wtPZ are from a previous study. ${ }^{14}$ 


\section{(A)}

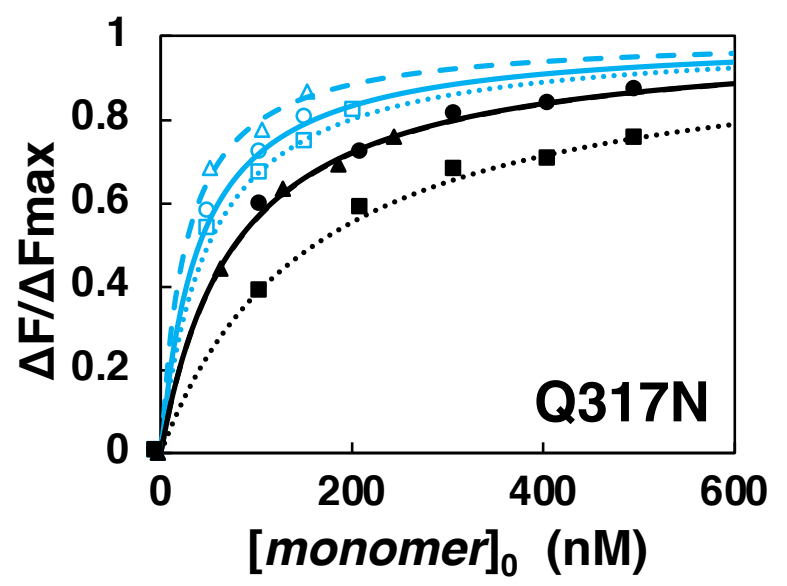

(D)

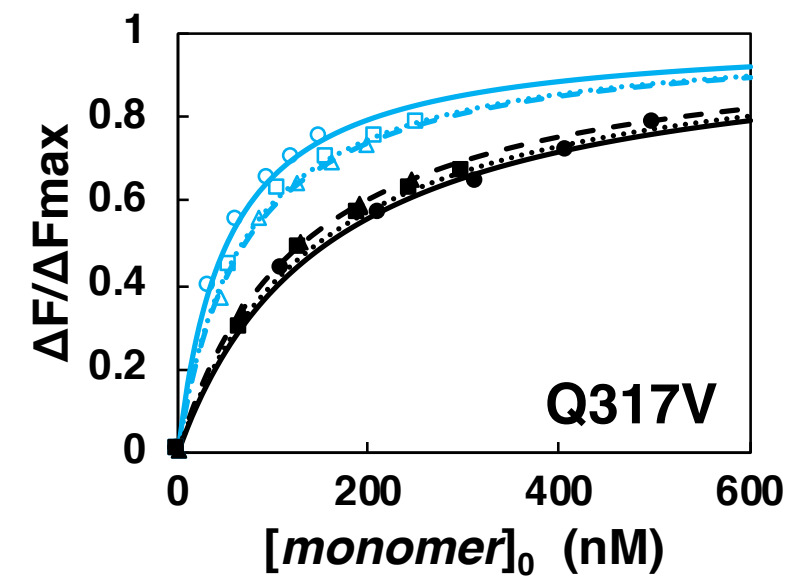

(B)

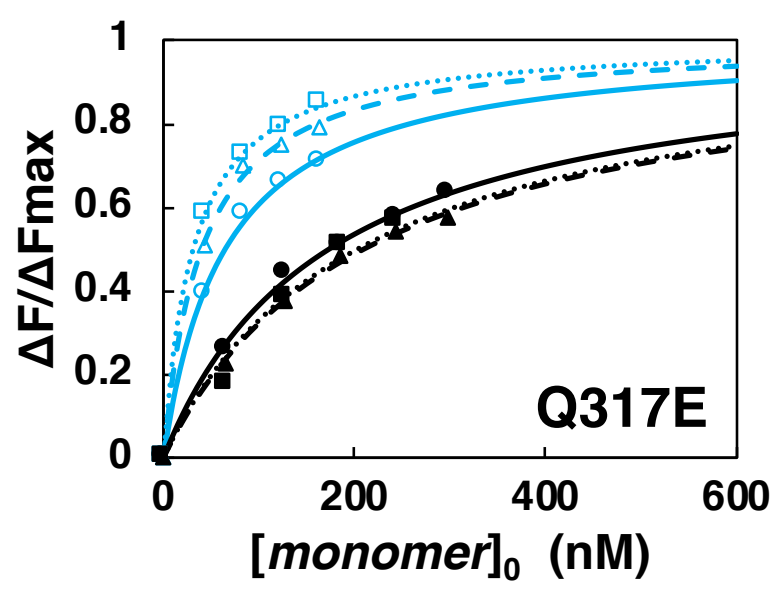

(C)

S11

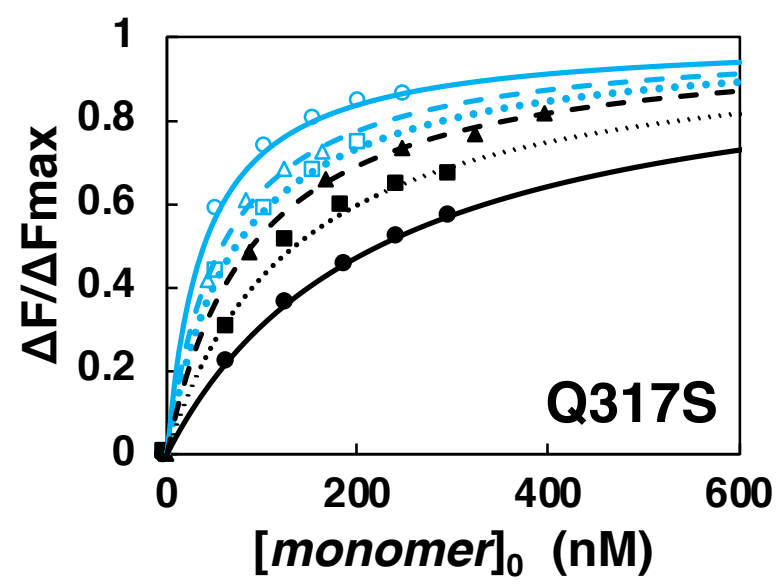

Fig. S9 Saturation binding behaviors $\left(\Delta F / \Delta F_{\max }\right)$ of [monomer $]_{0}$ of (A) Q317N, (B) Q317E, (C) Q317S, (D) Q317V, and (E) Q317L. Filled black circles and black lines indicate the D state, and open blue circles and blue lines indicate the L state. Measurements were performed more than 3 times in the $\mathrm{D}$ and $\mathrm{L}$ states. 


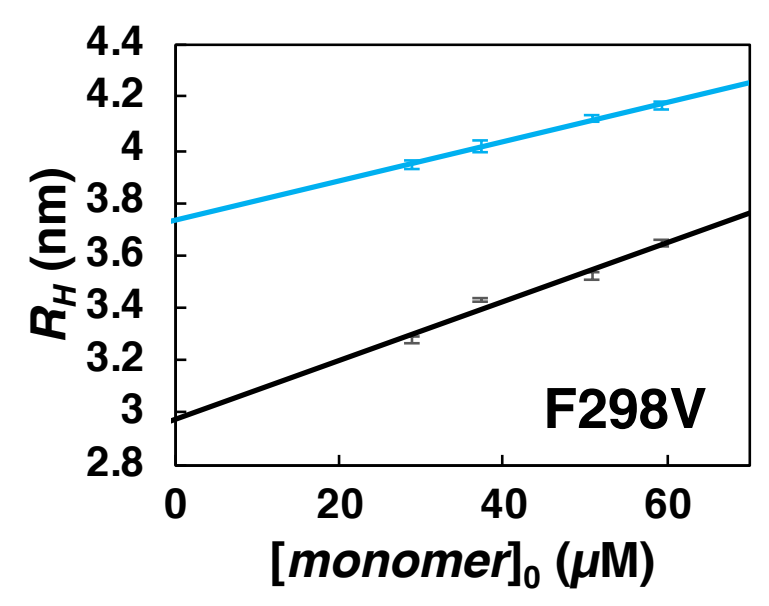

(C)

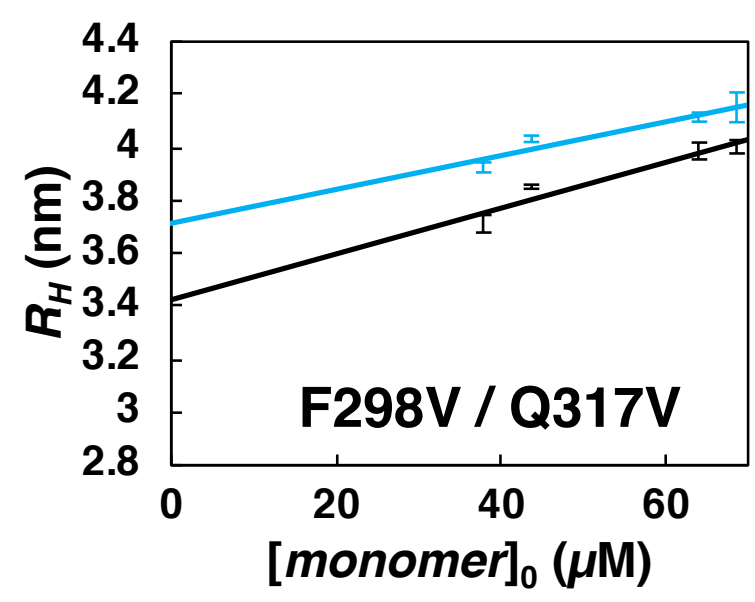

(D)

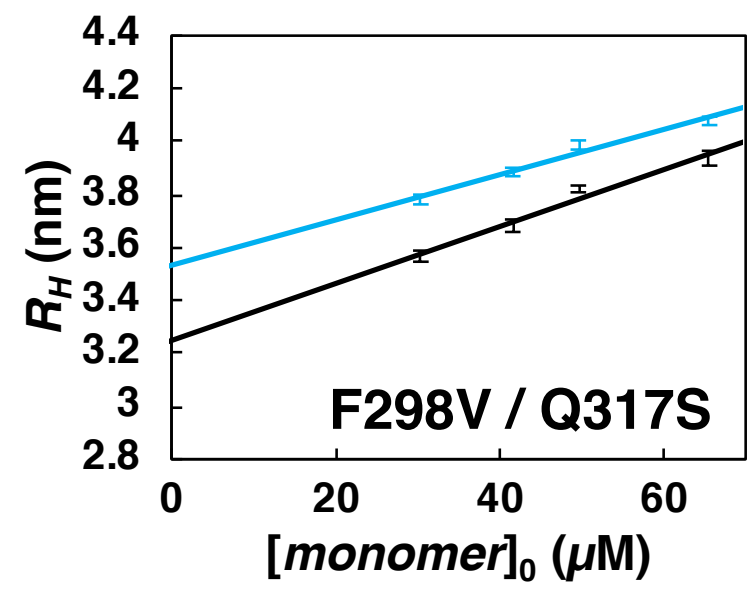

(E)

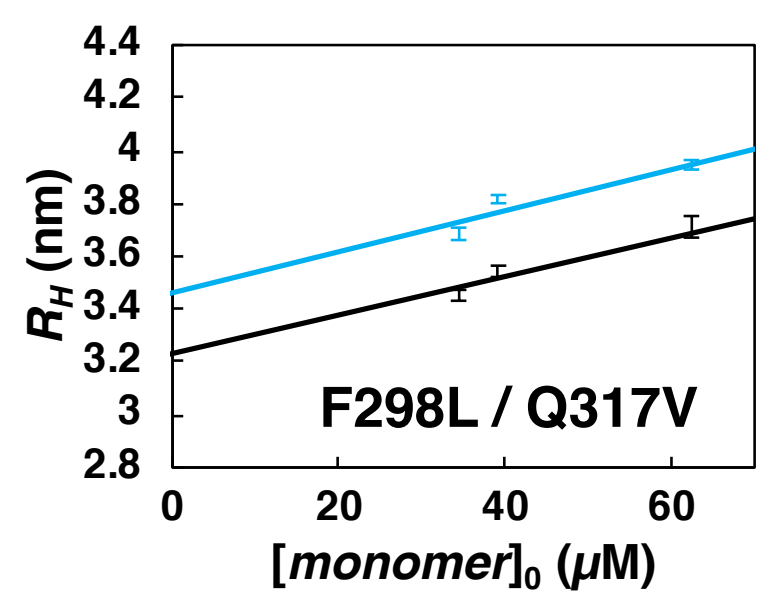

Fig. S11 The $R_{H(a p p)}$ according to protein concentration relative to [monomer] of (A) F298V, (B) F298L, (C) F298V/Q317V, (D) F298V/Q317S, and (E) F298L/Q317V. Black and blue symbols and lines represent the D and L states, respectively. Bars represent the $R_{H(a p p)}$ at each concentration with $\mathrm{SD}(\mathrm{n} \geq 6)$. 
(A)

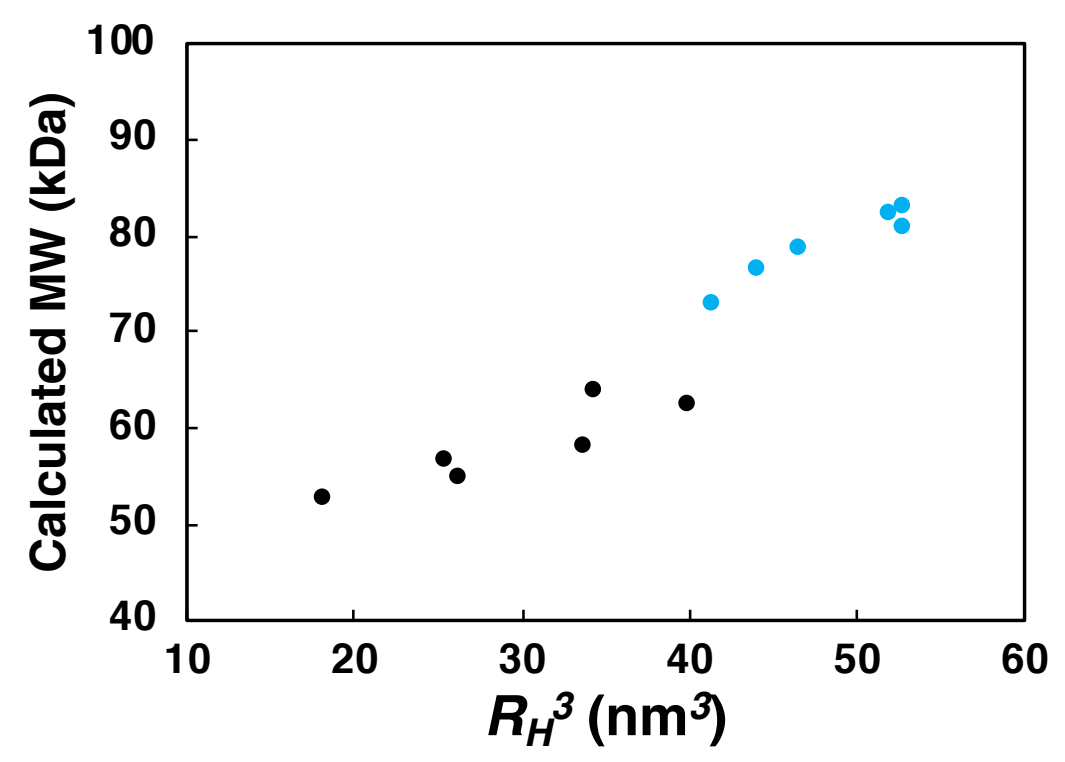

(B)

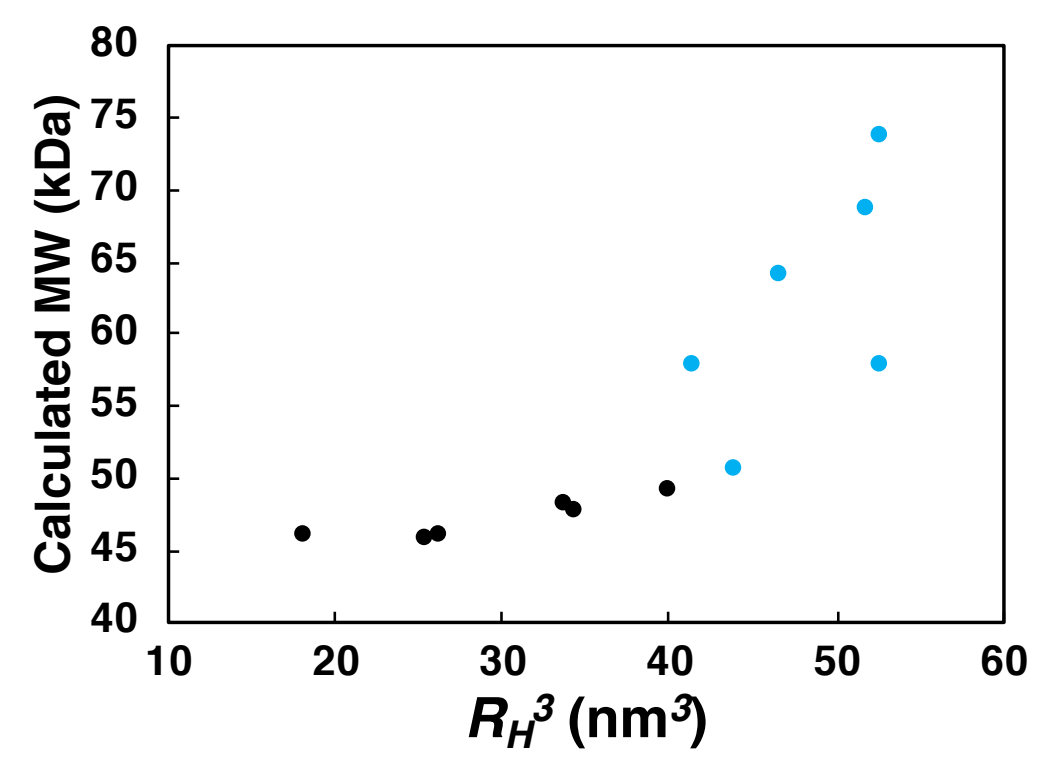

Fig. S12 Correlation between $R_{H}{ }^{3}$ and MW according to the peak elution volumes of F298 and F298/Q317 mutants and equation for globular proteins. ${ }^{23}$ Injected protein concentrations were (A) $100 \mu \mathrm{M}$ and (B) $8 \mu \mathrm{M}$. Black and blue symbols represent data in the D and L states, respectively. 
(A)

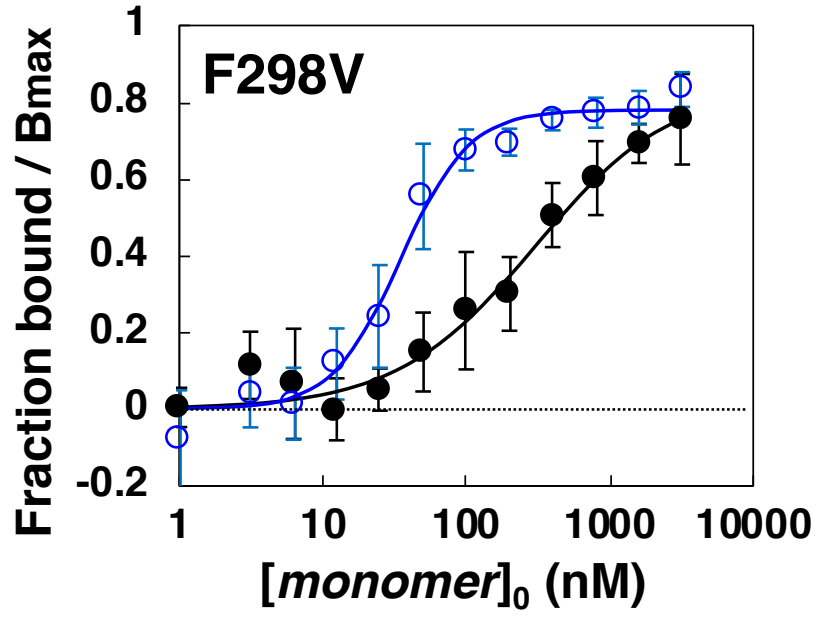

(C)

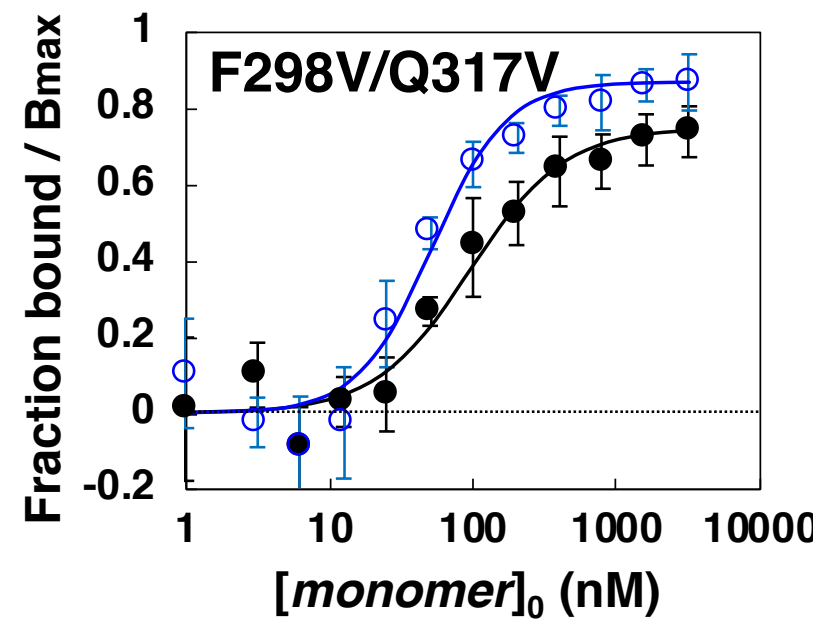

(B)

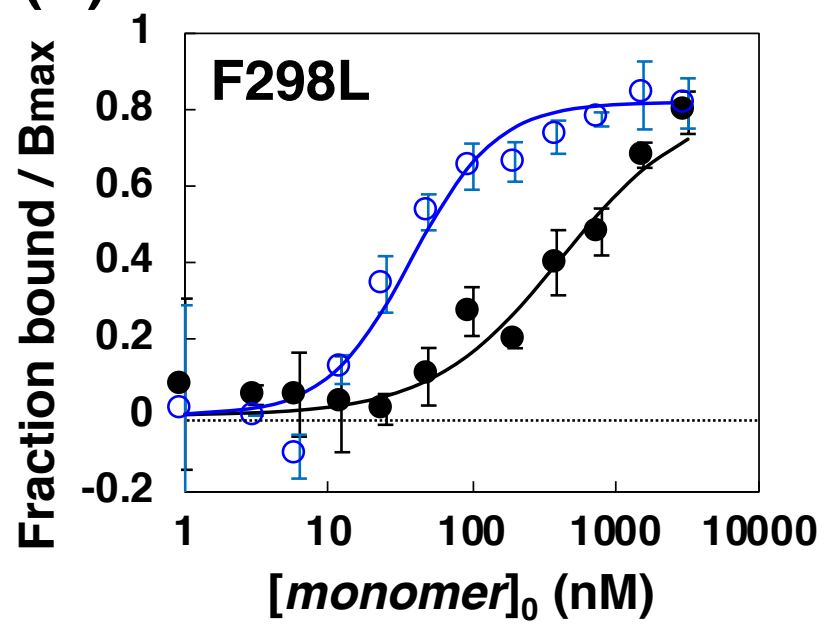

(E)
(D)

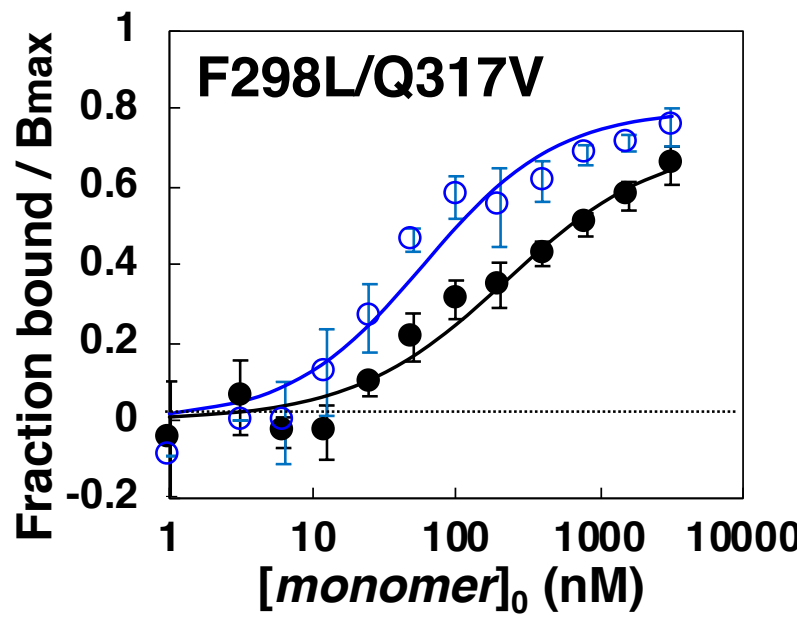

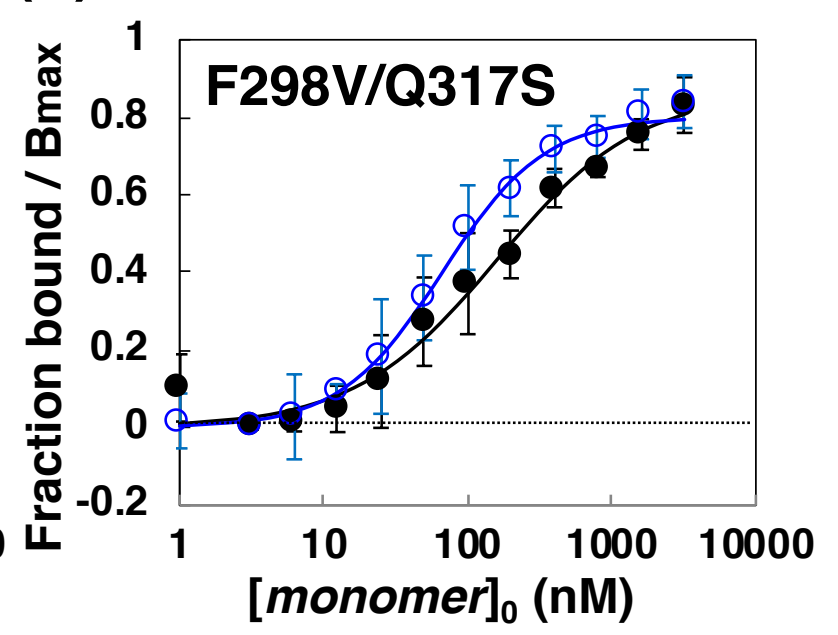

Fig. S13 The normalized dsApo fractions bound to the F298 and F298/Q317 mutants and plotted against protein concentrations [monomer $]_{0}$ with fitting curves in the $\mathrm{D}$ (filled black circles and black lines) and in the L (open blue circles and blue lines) states for (A) F298V, (B) F298L, (C) F298V/Q317V, (D) F298V/Q317S, and (E) F298L/Q317V. Bars represent SDs (n $\geq 4$ ). 


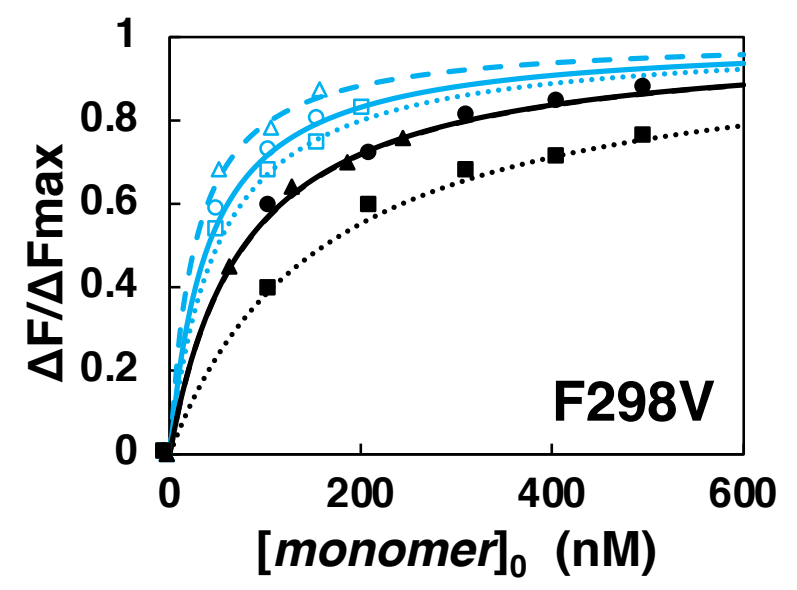

(C)

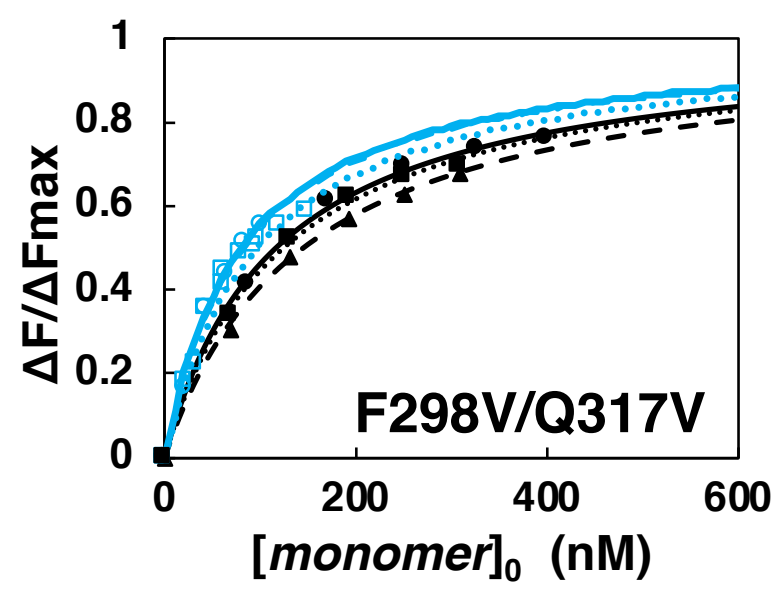

(D)

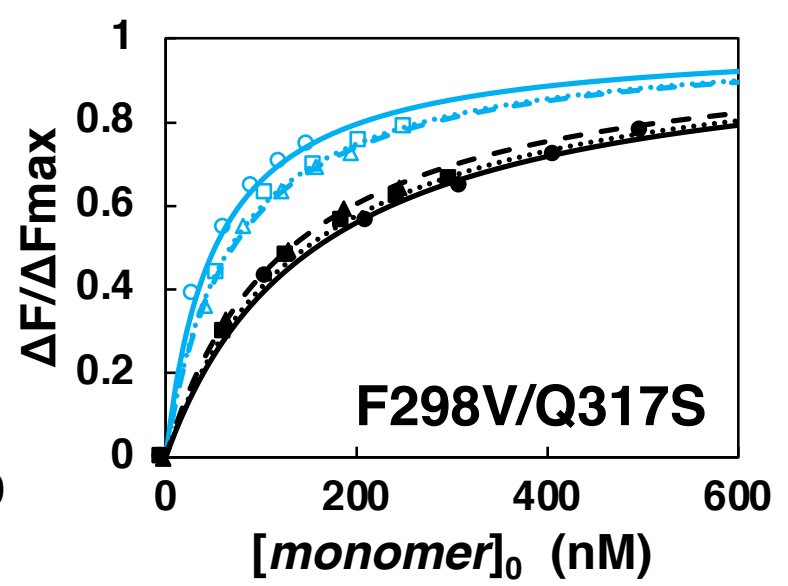

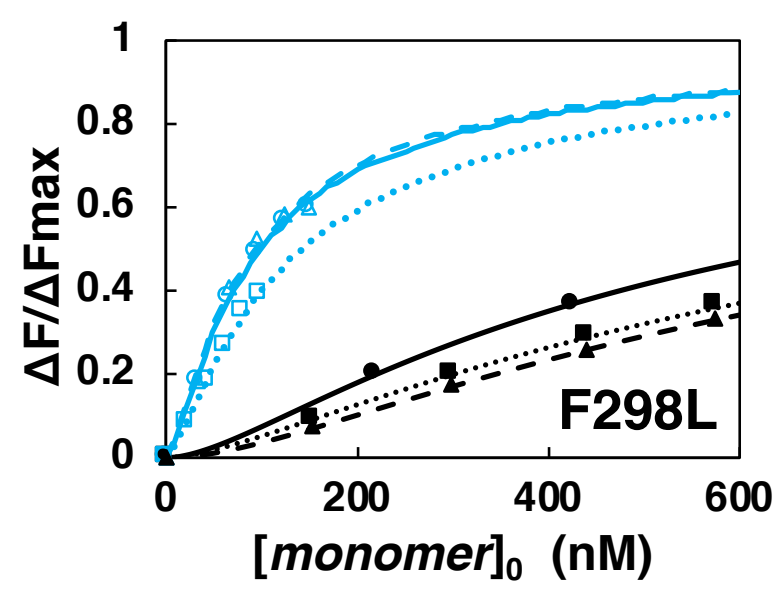

(E)

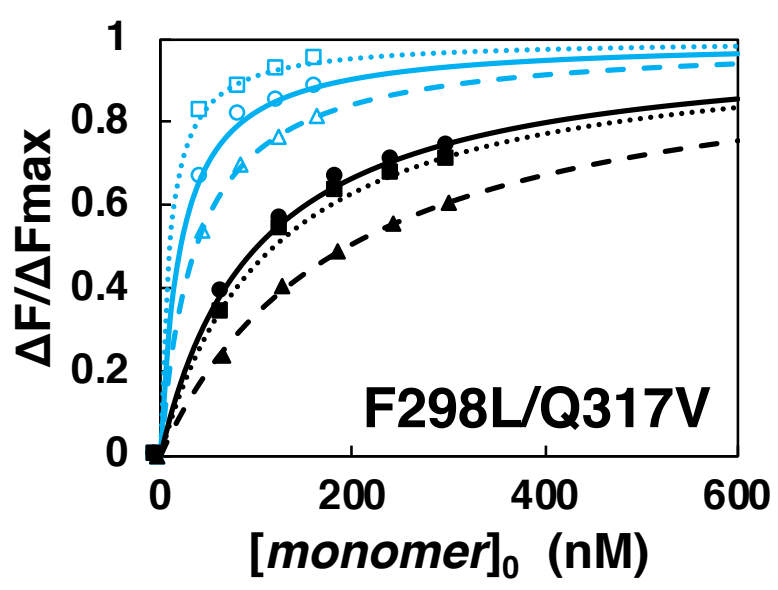

Fig. S14 Saturation binding behavior $\left(\Delta F / \Delta F_{\max }\right)$ against the [monomer $]_{0}$ of (A) F298V, (B) F298L, (C) F298V/Q317V, (D) F298V/Q317S, and (E) F298L/Q317V. Black symbols and lines indicate the $\mathrm{D}$ state, and blue symbols and lines indicate the $\mathrm{L}$ state. Measurements were performed more than 3 times in the $\mathrm{D}$ and $\mathrm{L}$ states. 
Table S1 Peak elution volumes and calculated MW of Q317 mutants

\begin{tabular}{|c|c|c|c|c|}
\hline & \multicolumn{2}{|c|}{$100 \mu \mathrm{M}$} & \multicolumn{2}{|c|}{$8 \mu \mathrm{M}$} \\
\hline & $\begin{array}{c}\text { D } \\
\text { mL (kDa) }\end{array}$ & $\mathrm{mL}^{\mathbf{L}}(\mathrm{kDa})$ & $\begin{array}{c}\text { D } \\
m L(k D a)\end{array}$ & $\stackrel{\mathrm{L}}{\mathrm{mL}(\mathrm{kDa})}$ \\
\hline wtPZ & $9.4(56.6)$ & $8.4(82.5)$ & $9.9(45.7)$ & $8.7(73.6)$ \\
\hline Q317N & $8.8(69.5)$ & $8.7(73.6)$ & $9.8(49.7)$ & $9.5(54.1)$ \\
\hline Q317E & $8.9(68.4)$ & $8.5(77.6)$ & $9.6(52.0)$ & $9.0(64.9)$ \\
\hline Q317S & $9.1(63.2)$ & 8.5 (77.9) & $9.9(46.6)$ & $9.2(59.7)$ \\
\hline Q317V & $9.1(62.5)$ & $8.4(81.2)$ & $9.8(48.4)$ & $8.7(71.6)$ \\
\hline Q317L & $9.1(62.7)$ & $8.4(79.4)$ & $9.9(47.0)$ & $8.9(67.7)$ \\
\hline
\end{tabular}


Table S2 Peak elution volumes and calculated MW of F298 and F298/Q317 mutants

\begin{tabular}{|c|c|c|c|c|}
\hline & \multicolumn{2}{|c|}{$100 \mu \mathrm{M}$} & \multicolumn{2}{|c|}{$8 \mu \mathrm{M}$} \\
\hline & $\begin{array}{c}\text { D } \\
m L(k D a)\end{array}$ & $\mathrm{mL}_{(\mathrm{kDa})}^{\mathrm{L}}$ & $\begin{array}{c}\text { D } \\
m L(k D a)\end{array}$ & $\underset{m L}{L}(k D a)$ \\
\hline wtPZ & $9.4(56.6)$ & $8.4(82.8)$ & $9.9(45.7)$ & $8.7(73.6)$ \\
\hline F298V & $9.4(54.9)$ & $8.4(82.1)$ & $9.8(45.9)$ & $8.8(68.7)$ \\
\hline F298L & $9.6(52.6)$ & $8.5(78.8)$ & $9.9(45.9)$ & $9.0(64.2)$ \\
\hline F298V / Q317V & $9.1(62.2)$ & $8.4(80.9)$ & $9.7(49.2)$ & $9.3(57.9)$ \\
\hline F298V / Q317S & $9.0(63.9)$ & $8.6(76.4)$ & $9.8(47.7)$ & $9.7(50.5)$ \\
\hline F298L / Q317V & $9.1(58.1)$ & $8.7(72.7)$ & $9.8(48.2)$ & $9.7(50.5)$ \\
\hline
\end{tabular}

